\title{
Wie fühlen sich Kopfschmerzen bei COVID-19 an?
}

Zu den möglichen Symptomen einer COVID-19-Erkrankung gehören auch Kopfschmerzen. Sie fallen nach den Angaben von erkranktem Medizinpersonal recht unterschiedlich aus, einige Gemeinsamkeiten scheint es aber zu geben.

\begin{abstract}
Laut Studien leiden 6-15\% der SARS-CoV-2-Infizierten an Kopfschmerzen. Kopfschmerzen gehören damit eher zu den Nebensymptomen („minor symptoms“) der Viruserkrankung. Aus Fallbeschreibungen geht jedoch hervor, dass Kopfschmerzen auch das erste Symptom von COVID-19 sein können. Spanische Neurologen haben deswegen versucht, spezifische Charakteristika von COVID-Kopfschmerzen herauszudestillieren. Dazu haben sie Mitarbeiter des Gesundheitssystems, die die Infektion selbst durchgemacht und dabei an Kopfschmerzen gelitten hatten, nachträglich zu ihren Beschwerden befragt.
\end{abstract}

\section{Das Wichtigste in Kürze}

Frage: Was charakterisiert COVID-19-assoziierte Kopfschmerzen? Antwort: Die Kopfschmerzsymptomatik ist sehr heterogen. Oft ist der Schmerz drückend und holo-, hemikraniell oder okzipital lokalisiert und nimmt bei Bewegung zu.

112 Personen beantworteten den Fragebogen, drei Viertel von ihnen hatten keine Kopfschmerzanamnese. Die COVID-assoziierten Kopfschmerzen traten in der Regel nicht zusammen mit Fieber auf, fast $90 \%$ der Befragten hatten Normaltemperatur. Im Mittel vergingen nach den ersten SARS-CoV-2-Symptomen wie Husten, Fieber und Muskelschmerzen bemerkbar machten.

\section{Drückende Schmerzen, die bei Bewegung zunehmen}

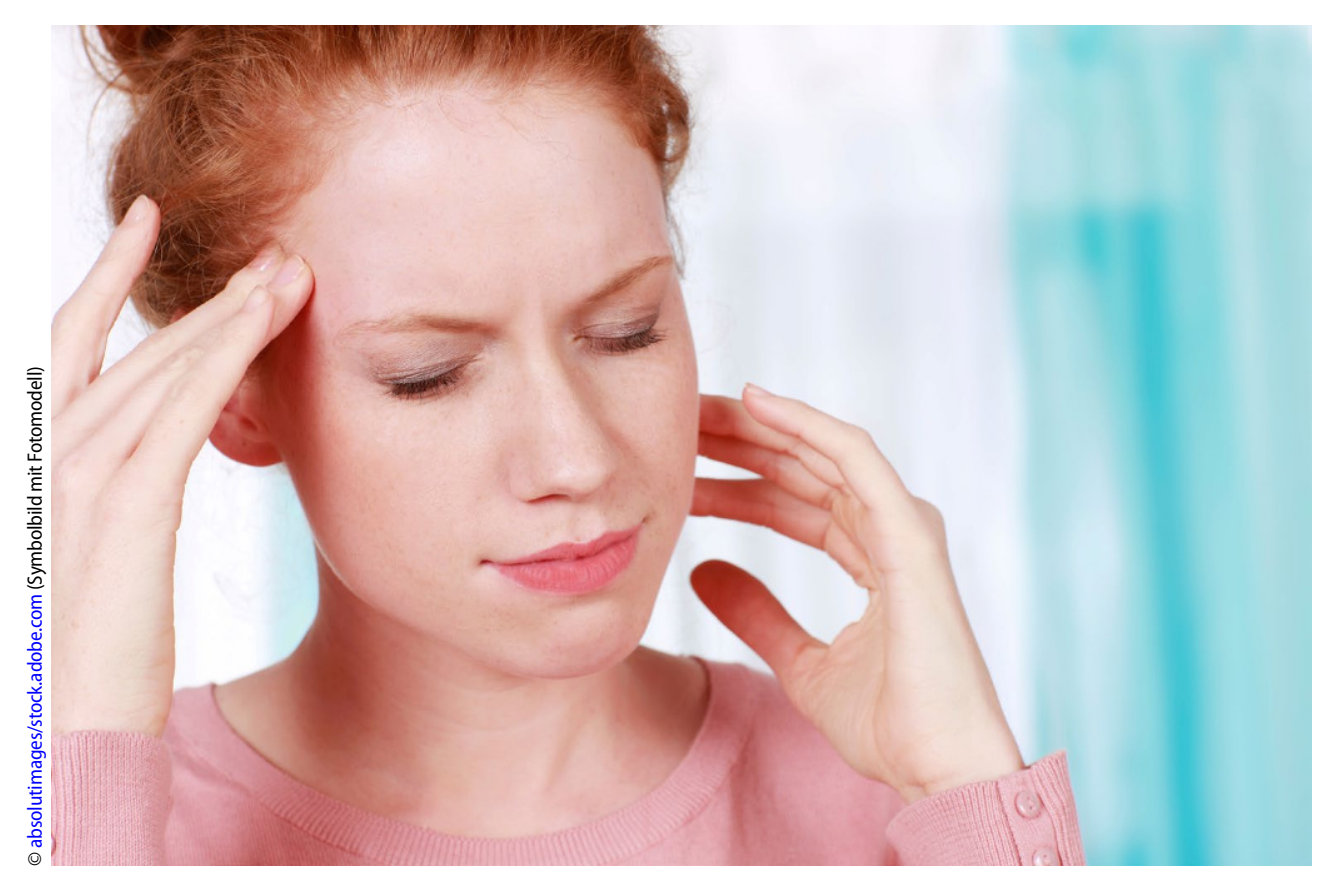

\ Frauen, die seltener als Männer eine schwere COVID-Erkrankung entwickeln, könnten häufiger von Kopfschmerzen betroffen sein rund drei Tage, bis sich Kopfschmerzen

Die Schmerzsymptomatik war, „bemerkenswert" heterogen, wie die Forscher um Jesus Porta-Etessam von der Universität in Madrid berichten. Am häufigsten waren sie hemi- oder holokraniell ( $46 \%$ bzw. $42 \%$ ), etwas seltener okzipital lokalisiert (18\%). Der Schmerzcharakter wurde meistens (zu $80 \%$ ) als drückend beschrieben. Pulsierende Schmerzen waren insgesamt selten (7\%), bei einer Migräneanamnese etwas häufiger (20\%). Körperliche Aktivität bzw. Kopfbewegungen führten bei etwa der Hälfte der Patienten zu einer Verstärkung der Schmerzen. Bei einem beträchtlichen Teil der Erkrankten waren die Kopfschmerzen außerdem mit Phono- oder Photophobie verbunden (41 \% bzw. $29 \%$ ). Unter Übelkeit litten weniger als $20 \%$. Über ein Drittel der Befragten vermuteten einen Zusammenhang zwischen den Kopfschmerzen und dem Tragen von Schutzmasken/-brillen.

Die Studienteilnehmer, zu $70 \%$ Ärzte, waren meist noch jünger und hatten daher überwiegend einen leichten bis mittelschweren COVIDVerlauf gehabt. Auffällig war der Frau- 
enanteil von rund $80 \%$ : Er könnte ein Hinweis sein, dass Frauen, die seltener als Männer eine schwere COVIDErkrankung entwickeln, häufiger von Kopfschmerzen betroffen sind.

\section{Vier Kopfschmerz-Typen}

Aufgrund der Heterogenität der beschriebenen Kopfschmerzcharakteristika postulieren die Studienautoren, dass es bei COVID-assoziierten Kopfschmerzen unterschiedliche Muster und Ursachen gibt. Sie schlagen eine Gruppierung in vier Typen vor:

1. Kopfschmerz in Zusammenhang mit dem Tragen von
Schutzmasken/-brillen, typischerweise drückender Schmerz, gehäuft bei Migränepatienten

2. Weniger spezifische, drückende Kopfschmerzen ohne Begleitsymptome

3. Kopfschmerzen bei Patienten mit primärer Kopfschmerzerkrankung, v.a. Migräne: Symptome häufig ähnlich wie in Vorgeschichte

4. Kopfschmerzen als Folge der SARS-CoV-2-Infektion: Verschlechterung durch Bewegung; häufig holo- oder hemikraniell; Begleitsymptom Phonophobie, seltener Photophobie, Übelkeit, Erbrechen
Hinweis des Verlags. Der Verlag bleibt in Hinblick auf geografische Zuordnungen und Gebietsbezeichnungen in veröffentlichten Karten und Institutsadressen neutral.

psychopraxis.neuropraxis2020-23:270-271 https://doi.org/10.1007/s00739020-00680-2

(c) Springer-Verlag GmbH Austria, ein Teil von Springer Nature 2020

\section{Studienanalyse}

\section{Warum Sonnenlicht gegen Multiple Sklerose hilft}

Die Sonne schützt vor Multipler Sklerose und mildert den Krankheitsverlauf - das ist schon lange bekannt. Es liegt aber nicht nur am Vitamin D. Forscher haben neue Gründe gefunden.

Der Zusammenhang ist frappierend und schon seit fast 100 Jahren bekannt: In polaren und gemäßigten Breiten mit wenig Sonne ist die MSInzidenz deutlich höher als in den Tropen und Subtropen, auch verläuft eine MS in sonnigen Regionen im Schnitt deutlich milder als in trüben Gefilden. Dieser Effekt lässt sich sogar für das relativ kleine Deutschland nachweisen, erläuterte Professor Heinz Wiendl von der Universität Münster auf dem virtuellen Kongress der Deutschen Gesellschaft für Neurologie (DGN).

\section{Schwere der MS korreliert mit Vitamin-D-Spiegeln}

Wiendl bezog sich auf eine Auswertung der deutschen MS-Kohorte mit rund 1400 Patienten. Danach haben Patienten in Norddeutschland einen schwereren Verlauf mit mehr Gadoli- nium-anreichernden Läsionen als Patienten im sonnigeren Süden.

Ein ähnlicher Effekt lässt sich auch in der französischen BioNat-MS-Kohorte beobachten. In beiden Kohorten korreliert die Schwere der MS zudem mit den Vitamin-D-Spiegeln bei der Aufnahme: Patienten mit den niedrigsten Werten zu Beginn entwickelten im Verlauf die stärksten Einschränkungen.

Auf einen kausalen Zusammenhang zwischen Vitamin D und MS deuten genetische Untersuchungen: Einzelnukleotid-Polymorphismen, die zu einer verminderten Vitamin-DProduktion führen, gehen mit einem zwei- bis vierfach erhöhten MS-Risiko einher.

Ist Vitamin D also tatsächlich der Hauptmediator für den Schutz durch Sonnenlicht? Dann sollte eine Supplementierung den MS-Verlauf deutlich bremsen. Das ist allerdings nur bedingt der Fall. In der randomisiertkontrollierten SOLAR-Studie mit 226 Patienten, die zu einer MS-Basistherapie mit Interferon beta-1a entweder hochdosiertes VitaminD3 oder Placebo bekamen, entwickelten Patienten mit der Vitaminsupplementierung ein Drittel weniger MRT-Läsionen und $30 \%$ weniger Schübe [1].

Allerdings war die Differenz bei den Schüben zur Placebogruppe nicht signifikant, ebenso wenig konnte die Therapie die Behinderungsprogression bremsen, was auch daran gelegen haben dürfte, dass sich der EDSS-Wert in beiden Gruppen kaum verändert hatte.

In kleineren Studien waren die Resultate gemischt: Manche fanden einen Nutzen, andere nicht. Wiendl verwies auf eine Cochrane-Analyse aus dem Jahr 2018: Hier zeigte sich über alle Studien gemittelt kein Effekt der
Quelle: www.

springermedizin.de/ Dr. Beate Schumache 